\title{
Characteristic Analysis and Experimental Verification of the Axially Asymmetric Structured Outer-Rotor Type Permanent Magnet Motor
}

\author{
Myung-Ki Seo*, Tae-Yong Lee*, Kyungsoo Park*, Yong-Jae Kim** and Sang-Yong Jung ${ }^{\dagger}$
}

\begin{abstract}
In this study, we have dealt with a design characteristic of outer-rotor type permanent magnet (PM) motor applied for Engine Cooling Fan (ECF). When we design a motor with structure like this type, it is required as a requisite to consider 3-Dimensional (3-D) effect by implementing a non-linear Finite Element Analysis (FEA) due to a yoke-ceiling, which is perpendicular to the axis of rotation. We have analyzed identical models under three different conditions. The analysis has been performed through a non-linear 2-Dimensional (2-D) and 3-D FEA. Finally, the results have been compared with Back Electro-Motive Force (BEMF) value of actual motor model. As a result, a yokeceiling function as an additional flux path and the operating point on B-H curve of rotor material is shifted to non-saturation region relatively. Accordingly, magnetic flux linkage can be increased and motor size can be decreased under same input condition to satisfy ECF specification, such as torque.
\end{abstract}

Keywords: Permanent Magnet, Yoke-ceiling, Flux linkage, Saturation, Back electro-motive force, Magnetic flux density

\section{Introduction}

The driving motor for Engine Cooling Fan (ECF) is usually designed as outer-rotor type since it has a definite advantage on rotation under constant speed with high inertia characteristic. Accordingly, the motors which have this structure have been used commonly in low-power and constant speed rotation applications including fan, pump, and disk drive. In addition, the power density of outer-rotor type motor is relatively greater than that of inner-rotor type since the rotor diameter of outer-rotor type is larger than that of inner-rotor type. Structurally, the rotor is generally greater than the axial length of the magnets and limits axial length simultaneously. It permits the "additional flux linkage" along the yoke-ceiling, since the permanent magnets are attached to the inside of a deep-drawn rotor. The increase of flux linkage leads to reduction of the overall size and a yoke thickness. Fig. 1 shows the structure of outer-rotor type ECF motor.

The characteristics and torque ripple reduction in outerrotor type Permanent Magnet (PM) motor have been addressed in respect of unbalanced magnetic force (UMF). However, few works have been published in terms of additional magnetic flux linkage resulting from yokeceiling. Jang and Yoon [1] introduce that axially asymmetric PM motor generate axial UMF and it causes torque ripple. Jang and Kim [2] expressed method for

$\dagger$ Corresponding Author: School of Electronic and Electrical Engineering, Sungkyunkwan University, Korea. (syjung@skku.edu)

* School of Electronic and Electrical Engineering, Sungkyunkwan University, Korea. (\{audr16277, ty.lee\}@skku.edu)

* Dept. of Electrical Engineering, Chosun University, Korea. (kimyj21@chosun.ac.kr)

Received: September 4, 2015; Accepted: December 25, 2015 torque ripple reduction resulting from UMF through asymmetry of the permanent magnet with respect to the stator. However, these researches are not based on magnetic flux linkage but based on only magnetic force.

In this paper, the importance of 3-Dimensional (3-D) Finite Element Analysis (FEA) in terms of magnetic flux linkage for designing outer-rotor type motor considering the yoke-ceiling has been studied. Accordingly, we have analyzed identical models under three different conditions. One is 2-Dimensional (2-D) analysis model. Another is 3$\mathrm{D}$ analysis model without considering the yoke-ceiling.

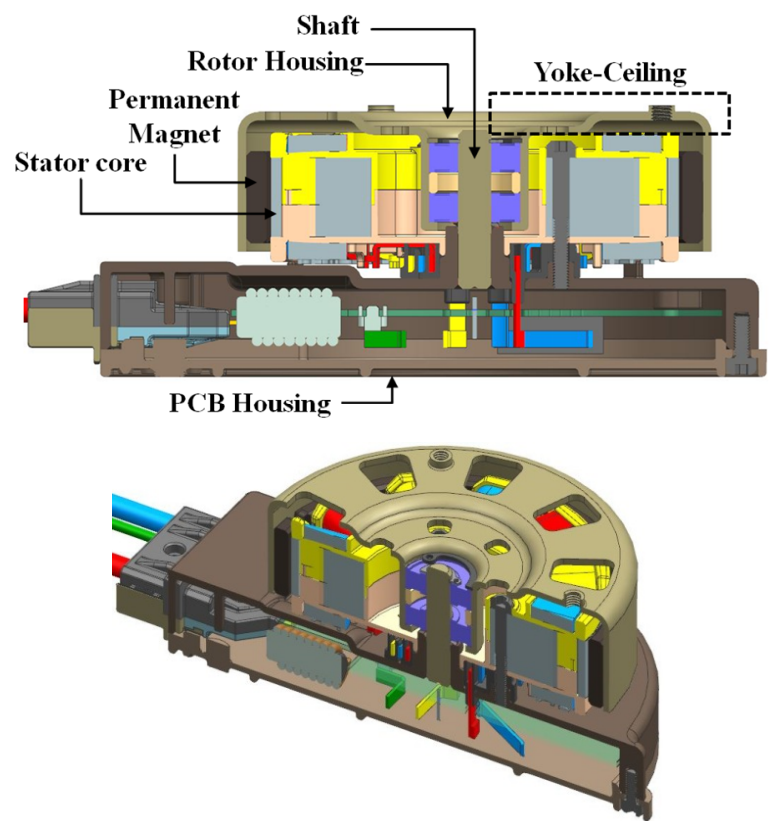

Fig. 1. Structure of outer-rotor type ECF motor. 
The other is 3-D analysis one considering whole parts. The analyses have been performed through a non-linear FEA. Finally, the results have been compared with experimental results of actual motor model.

\section{Analysis Model}

The motor dealt in this study is applied to an ECF powered by a $12 \mathrm{~V}$ battery. The rating output power, speed, and torque of this motor is 320 [W], 2,500 [r/min], and $1.23[\mathrm{Nm}]$, respectively. The specification of this model is shown in Table 1.

Table 1. Specification of a reference model.

\begin{tabular}{c|c|c|c}
\hline Classification & Design Parameter & Specification & Unit \\
\hline \multirow{2}{*}{ Performance } & Torque & 1.23 & {$[\mathrm{Nm}]$} \\
\cline { 2 - 4 } & Speed & 2,500 & {$[\mathrm{r} / \mathrm{min}]$} \\
\hline General & Outer Diameter & 115 & {$[\mathrm{~mm}]$} \\
\hline \multirow{2}{*}{ Stator } & Poles & 8 & \\
\cline { 2 - 4 } & Slots & 12 & \\
\hline Magnet & Remanence of Magnet & 0.44 (Ferrite) & {$[\mathrm{T}]$} \\
\hline
\end{tabular}

\section{Effect of Yoke-Ceiling}

\subsection{Magnetic flux distribution}

The main difference between 2-D and 3-D model is whether or not to consider the yoke-ceiling, which is a perpendicular to the axis of rotation. In addition, the magnetic flux distribution also becomes different in consequence of it. Fig. 2 shows the magnetic flux line

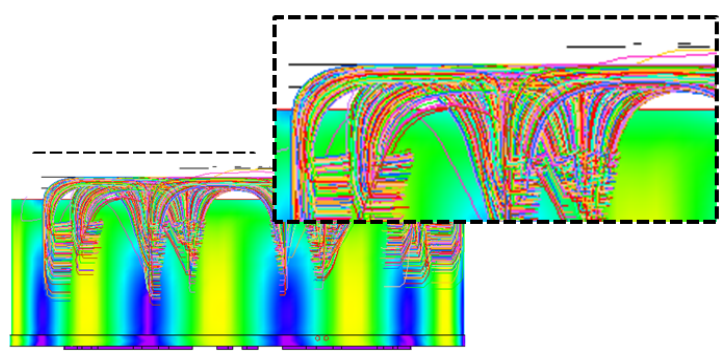

(a)

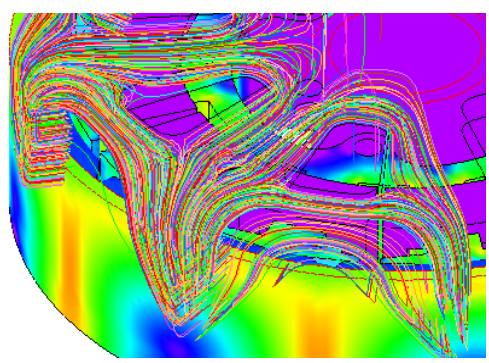

(b)

Fig. 2. Magnetic flux flowing the yoke-ceiling in 3-D model: (a) Left side; (b) Oblique side. flowing along the yoke-ceiling according to several angles in the 3-D model. As shown for the 3-D model in Fig. 2, the magnetic flux is distributed to not only the back-yoke but also the proximity of the yoke-ceiling in contrast with 2-D model since that part is used as additional magnetic flux path.

In 2-D model, the magnetic flux spreading along the yoke-ceiling is not considered, while it is taken into consideration in 3-D model. In other words, it is impossible to represent the yoke-ceiling in a 2-D FEA, leading to a difference between the results of the 2-D and 3-D models. For this reason, it results in the specific and overall differences such as teeth width and power density, since the amount of flux linkage passing through the rotor in 2-D model is different from 3-D model.

The difference of the portion which is used as a magnetic flux path such as teeth and inner yoke is associated with a flux linkage amount. As noted above, in 2-D model, the magnetic flux flowing along the rotor and stator is less than it in 3-D model, since the yoke-ceiling is not considered. Accordingly, widths of teeth and inner yoke are designed smaller in 2-D model compared to 3-D model. If we make prototype and carry out performance test without considering the additional magnetic flux passing through the yoke-ceiling, the unintended effect of the extended magnetic flux path resulting from yoke-ceiling is applied to analysis model. As a result, the operating point of rotor and stator are moved to non-saturation and saturation region respectively, and the magnetic flux linkage is increased. Accordingly, the machine does not operate normally, since the magnetic flux linkage and BEMF are increased beyond limited input condition.

Fig. 3 (a), (b), and (c) show the three kinds of analysis models, respectively. (a) is 2-D analysis model, (b) is 3-D analysis model without considering the yoke-ceiling, and lastly (c) is 3-D analysis one considering whole parts. As mentioned previously, the widths of teeth and inner yoke of (a) and (b) are less than (c). Widths of teeth and inner-yoke are $3.0 \mathrm{~mm}$ and $6.0 \mathrm{~mm}$ each in model 1 and 2 , and that of model 3 are $4.5 \mathrm{~mm}$ and $8.0 \mathrm{~mm}$. Each width is minimum value for non-saturation operation. The differences between
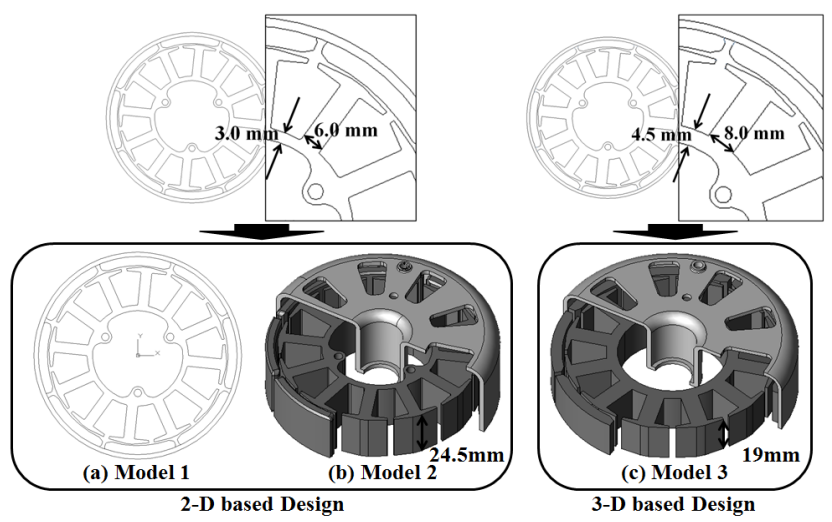

Fig. 3. Structure of analysis model. 
Table 2. Characteristics of analysis models.

\begin{tabular}{c|c|c|c}
\hline Classification & Model 1 & Model 2 & Model 3 \\
\hline Design & 2-D & 2-D & 3-D \\
\hline Analysis & 2-D & 3-D & 3-D \\
\hline Yoke-ceiling & $\mathrm{X}$ & O & O \\
\hline Stack [mm] & 24.5 & 24.5 & 19.0 \\
\hline Teeth [mm] & 6.0 & 6.0 & 8.0 \\
\hline Inner-yoke [mm] & 3.0 & 3.0 & 4.5 \\
\hline
\end{tabular}

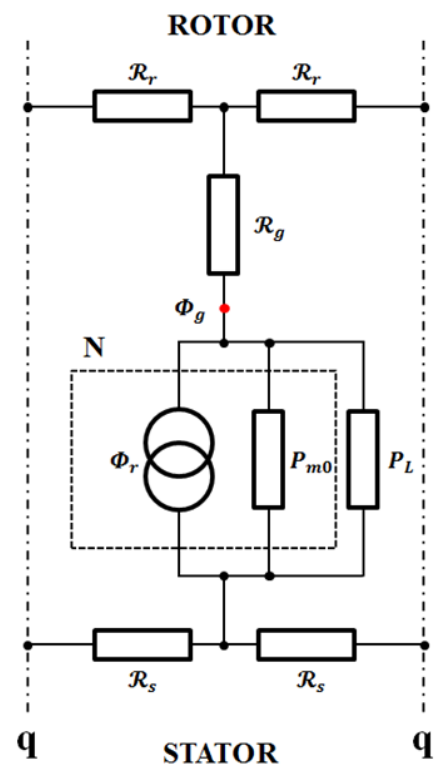

Fig. 4. Magnetic circuit for one pole.

each model are summarized in Table 2.

\subsection{Perspective of magnetic circuit}

The magnetic equivalent circuit for one pole is in Fig. 4. The airgap flux per pole $\Phi_{g}$ is driven through the airgap reluctance $\mathcal{R}_{g} . \Phi_{r}$ means remanent flux which is the product of remanent flux density $\left(B_{r}\right)$ and surface area of PM $\left(A_{m}\right) . \mathcal{R}_{r}$ and $\mathcal{R}_{s}$ represent reluctance of rotor and stator. All these parameters vary with the dimensions and material properties. Under no-load conditions it is usually adequate to consider only one pole, and to make use of symmetry. Accordingly, in Fig. 4 the quadrature axes q-q can be assigned zero magnetic potential. From Fig. 4, the flux through the magnet is in (1)

$$
\Phi_{m}=\Phi_{g}+\Phi_{L}=\Phi_{g}+\frac{P_{L}}{P_{m 0}+P_{L}}\left(\Phi_{r}-\Phi_{g}\right)
$$

where $\Phi_{m}$ is the flux through the magnet and $\Phi_{L}$ is the leakage flux. $P_{L}$ and $P_{m 0}$ represent internal magnet permeance and leakage permeance respectively. In addition, the sum of these factors means magnet permeance $P_{m}$ in (2).

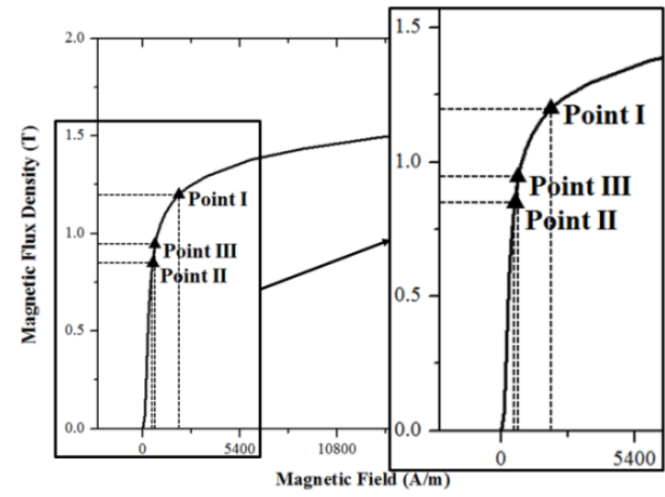

Fig. 5. B-H curve of rotor core and operating points of each model.

$$
P_{m}=P_{m 0}+P_{L}
$$

Meanwhile, when the airgap and the rest reluctance of the magnetic circuit can be represented by a lumped external reluctance $\mathcal{R}_{g}{ }^{\prime}$ in (3), the airgap flux is given by (4).

$$
\begin{gathered}
\mathcal{R}_{g}{ }^{\prime}=\mathcal{R}_{g}+\frac{\mathcal{R}_{r}+\mathcal{R}_{s}}{2} \\
\Phi_{g}=\frac{1 / \mathcal{R}_{g}{ }^{\prime}}{1 / \mathcal{R}_{g}{ }^{\prime}+P_{m 0}+P_{L}} \Phi_{r}=\frac{1}{1+P_{m} \mathcal{R}_{g}{ }^{\prime}} \Phi_{r}
\end{gathered}
$$

Eqs. (1)-(4) are characteristic equations of magnetic system, and are introduced to account for difference of the magnetic flux path and flux linkage in models mentioned above. Meanwhile, Fig. 5 represents B-H curve of the rotor material in analysis model, and points I, II, and III are operating points of (a), (b), and (c) in Fig. 3. The y-values of these points are the Root Mean Square (RMS) values of the magnetic flux density at all nodes in FEA, and we assume that these are representative values of the magnetic flux density at each operation points. In Fig. 5, points II and III are in non-saturation region relatively while point I is in saturation region according to each magnetic flux density. Therefore, rotor reluctance $\left(\mathcal{R}_{r}\right)$ of point I is greater than that of Point II and III relatively. Lastly, according to (3) and (4), airgap flux per pole $\left(\Phi_{g}\right)$ of model considering yoke-ceiling is higher than model without considering it. This is result from the function of the yoke-ceiling, which is the part used for additional magnetic flux flowing along the rotor.

\section{Finite Element Analysis of BEMF}

Models were developed in FEA to the topologies 1, 2 and 3. In model 1, 2-D FEA is conducted, as not considering yoke-ceiling. In model 2, 3-D FEA is conducted in a same condition as in model 2, except addition of yoke-ceiling. Lastly, in model 3, 3-D FEA is conducted, considering 
Table 3. Coil flux linkage and BEMF of FEA.

\begin{tabular}{c|c|c|c|c}
\hline Classification & Model1 & Model2 & Model3 & Unit \\
\hline Flux linkage & 0.007587 & 0.012716 & 0.008774 & {$[\mathrm{~Wb}]$} \\
\hline BEMF & 5.79 & 9.76 & 6.67 & {$\left[\mathrm{~V}_{\mathrm{rms}}\right]$} \\
\hline
\end{tabular}

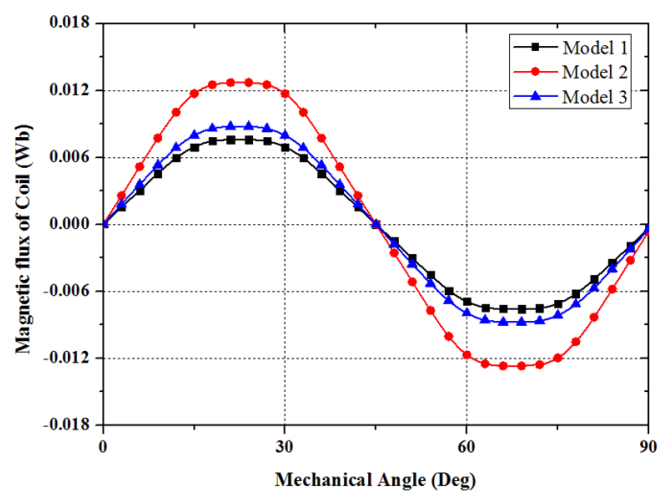

(a)

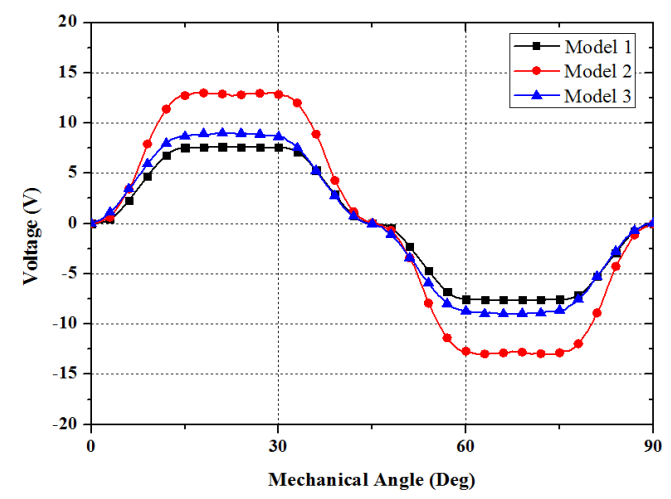

(b)

Fig. 6. Coil flux linkages and BEMFs of FEA.

whole part from the beginning of design process. Model 1 has 18884 triangular elements and models 2, 3 have 376621,319812 tetrahedral elements each; the number of elements in 3-D FEA models is even more than that of 2-D FEA model. The difference in terms of stack length is caused by more or less calculated flux linkage, according to whether the yoke-ceiling is considered in design process. Fig. 6 shows coil flux linkage and BEMF, and Fig. 7 shows magnetic flux density of each models. In the Fig. 6, the magnetic flux density of teeth and inner yoke in model 2 is higher than that of model 1. It is due to the additional unintended flux path, which results in more magnetic flux than expected. Table 3 shows numerical value of coil flux linkage and BEMF. These values are computed at rated speed. As can be seen from the Table 3, whole models show a difference in the coil flux linkage amount, whereby 0.007587 [Wb], 0.012716 [Wb], and 0.008774 [Wb] correspond to the 2-D and 3-D models, respectively for the proposed design. The coil flux linkage of the model 2 is $167.6[\%]$ of that of the model 1 . This discrepancy is due to

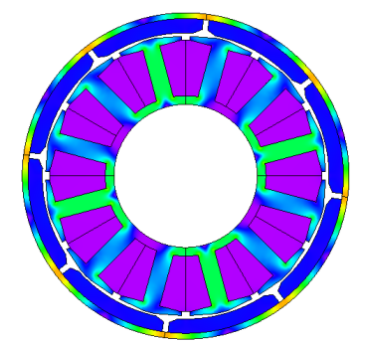

(a) Model1

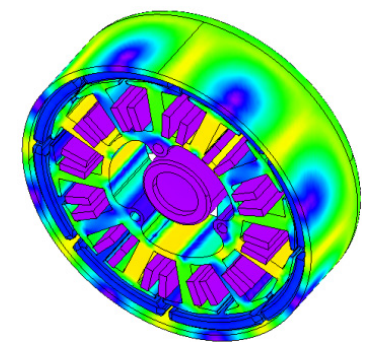

(b) Model2
Fig. 7. Magnetic flux density of each model.

the additional flux path that is obtained in a yoke-ceiling, and the numerical value is the basis of the BEMF and power density differences.

\section{Experimental Measurement of BEMF}

After considering the difference between the amounts of flux linkage in each model through FEA, the experimental measurement is carried out on models 2 and 3. BEMF is measured to figure out the influence of additional flux path; the test is conducted according to the yoke-ceiling effect. Universally, the increase of flux linkage is

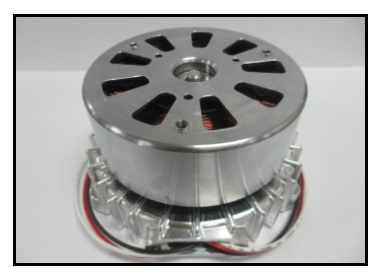

(a) Model 2

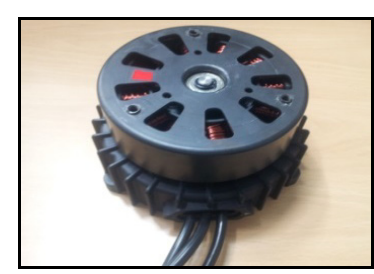

(b) Model 3
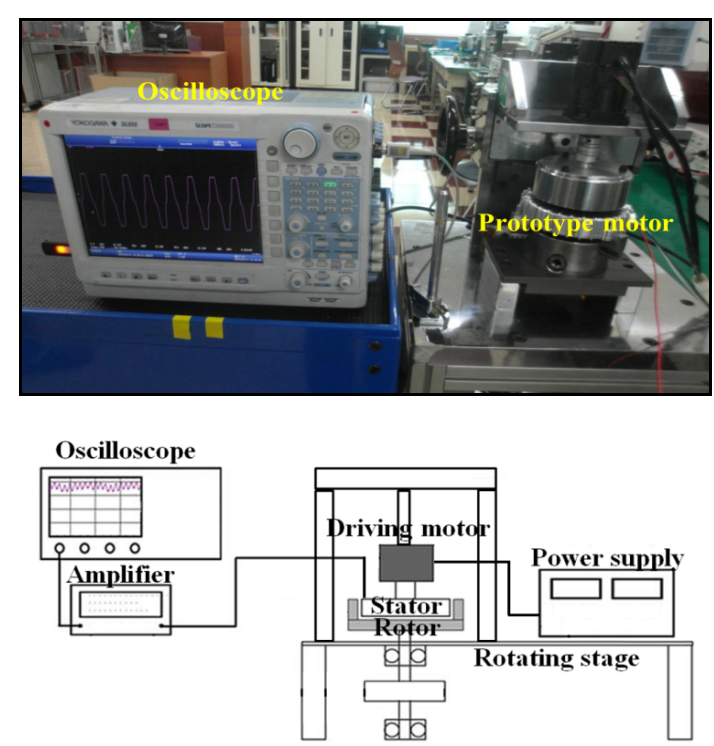

(b) Experimental setup

Fig. 8. Prototype motors and experimental setup. 
Table 4. BEMFs of experimental measurement.

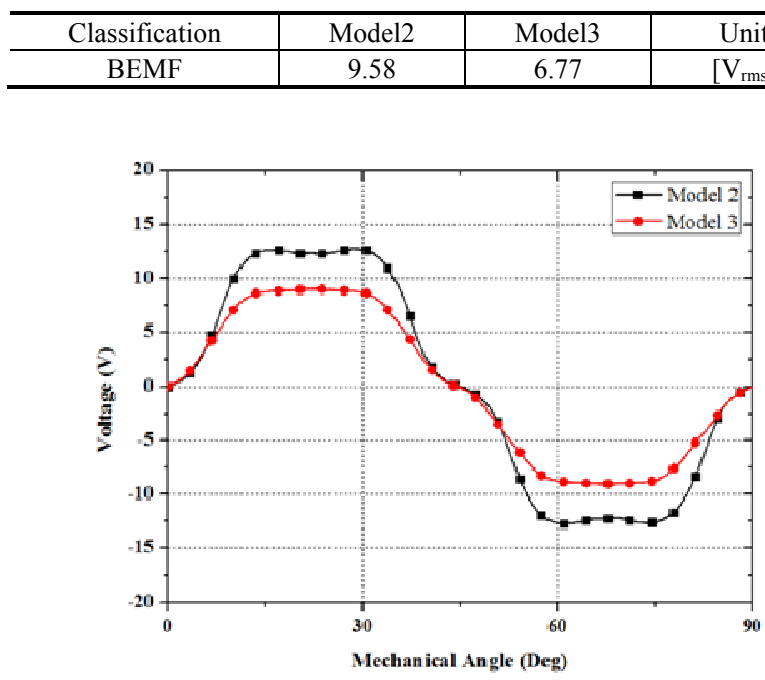

Fig. 9. BEMFs of experimental measurement

confirmed by measurement of BEMF indirectly, since the flux linkage is proportional to BEMF. Fig. 8 shows the structure of prototype motor and experimental setup for measurement of BEMF. The experimental setup consists of oscilloscope, prototype motor, and rotating stage. Fig. 9 shows BEMF measured in this experiment. The results are shown in Table 4. The BEMF of model 2 and 3 in experiment are $9.58 \mathrm{~V}_{\mathrm{rms}}$ and $6.77 \mathrm{~V}_{\mathrm{rms}}$ each, while the values are $9.76 \mathrm{~V}_{\mathrm{rms}}$ and $6.67 \mathrm{~V}_{\mathrm{rms}}$ in FEA. The error rates are $1.8 \%$ and $1.5 \%$ each. This results show the validity of FEA result and influence of the yoke-ceiling in this model, which has outer-rotor structure.

\section{Results and Discussion}

As a result, model 3 is the final model that considers influences mentioned above on the yoke-ceiling. The results of FEA and performance test are shown in Fig. 10 and table .05. Torque and cogging torque are valid in accordance with analysis and test results. Model 1 which is 2-D based design is analyzed using 2-D FEA, and model 2 which is 2-D based design is analyzed with 3-D FEA; only the yoke-ceiling is added. Lastly, 3-D based design, model 3 is analyzed through 3-D FEA; whole parts are considered in design process. In magnetic circuit having this structure, the yoke-ceiling is used as additional flux path, and it transforms magnetic flux distribution in stator and rotor.

Consequentially, teeth and inner yoke have to be designed wider than 2-D based design, since more magnetic flux has to flow. In addition, stack length must be designed shorter than 2-D based design, since BEMF increases as the analogy of flux linkage increase. BEMF and flux linkages in model 1 and 2 are different from that of model 3, since the yoke-ceiling is not considered; it is confirmed through
Table 5. Analysis and performance test result of model 3.

\begin{tabular}{c|c|c|c}
\hline Classification & 3-D FEA & Performance test & Unit \\
\hline BEMF & 6.67 & 6.77 & {$[\mathrm{~V}]$} \\
\hline Torque & 1.23 & 1.22 & \multirow{2}{*}[\mathrm{Nm}]{} \\
\hline Cogging Torque & 0.054 & 0.070 & \\
\hline
\end{tabular}

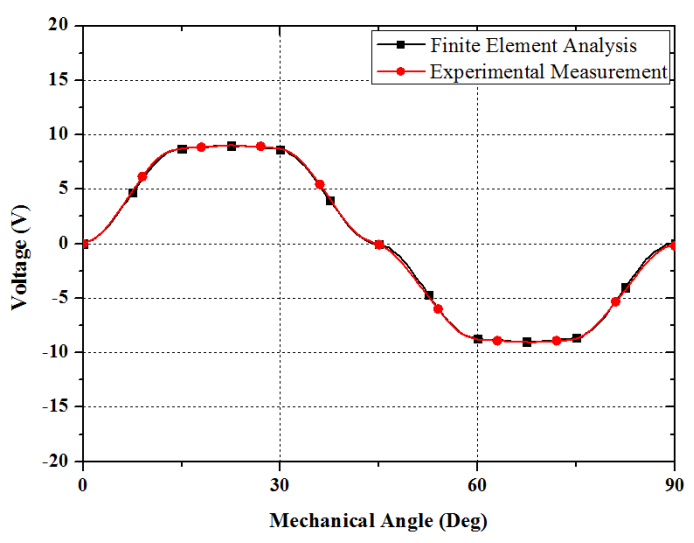

Fig. 10. Comparison of FEA and experimental measurement about BEMF.

experimental measurement. In conclusion, when we design outer-rotor type electric machine, the additional flux path spreading out beyond the permanent magnet in the rotor must be considered to determine specific size, such as teeth width or stack length.

\section{Conclusion}

In this paper, the influence of additional magnetic flux path is dealt with. The additional flux path enables more magnetic flux to flow along the rotor core, decreases the size, and increases BEMF in terms of performance. Accordingly, it is essential to conduct 3-D design process, when designing outer-rotor type motor with these advantages. It is way of apply influence of additional flux path, which is not considered in 2-D. It is main factor that decide specific size such as teeth width and stack length. Thus, in this paper, FEA and experimental measurement are conducted on analysis models in three different conditions to confirm the effect of additional flux path. Consequentially, the yokeceiling must be considered when designing motor having outer-rotor structure such as fan and blower.

\section{Acknowledgements}

This work was supported by the Human Resources Development program (No.20134010200550) of the Korea Institute of Energy Technology Evaluation and Planning (KETEP) and by the Basic Science Research Program through the National Research Foundation of Korea (NRF) 
funded by the Ministry of Education, Science and Technology under Grant NRF-2013R1A1A1A05011966.

\section{References}

[1] C. I. Lee, and C. H. Jang, "Experimental Measurement and Simulated Verification of the Unbalanced Magnetic Force in Brushless DC Motors," IEEE Trans. Magn., vol. 44, no. 11, pp. 4377-4380, Nov. 2008.

[2] G. H. Jang, J.W. Yoon, N. Y. Park, and S. M. Jang, "Torque and Unbalanced Magnetic Force in a Rotational Unsymmetric Brushless DC Motors" IEEE Tans. Magn., vol. 32, no. 5, pp. 5157-5159, Sep. 1996.

[3] Yon-Do Chun, Ju Lee, and Shinji Wakao, "Overhang Effect Analysis of Brushless DC Motor by 3-D Equivalent Magnetic Circuit Network Method," IEEE Trans. Magn., vol. 39, no. 3, pp. 1610-1613, May. 2003.

[4] Jung-Moo Seo, In-Soung Jung, Hyun-Kyo Jung, and Jong-Suk Ro, "Analysis of Overhang Effect for a Surface-Mounted Permanent Magnet Machine Using a Lumped Magnetic Circuit Model," IEEE Trans. Magn., vol. 50, no. 5, pp. 1119-1123, May. 2014.

[5] J. Y. Kim, S. J. Sung, and G. H. Jang, "Characterization and Experimental Verification of the Axial Unbalanced Magnetic Force in Brushless DC Motors," IEEE Trans. Magn., vol. 48, no. 11, pp. 3001-3004, Nov. 2012.

[6] S. J. Sung, G. H. Jang, J. W. Jang, J. Y. Song, and H. J. Lee, "Vibration and Noise in a HDD Spindle Motor Arising from the Axial UMF Ripple," IEEE Trans. Magn., vol. 49, no. 6, pp. 2489-2494, Jun. 2013.

[7] Andrej Burakov, and Antero Arkkio, "Comparison of the Unbalanced Magnetic Pull Mitigation by the Parallel Paths in the Stator and Rotor Windings," IEEE Trans. Magn., vol. 43, no.12, pp. 4083-4088, Dec. 2007.

[8] G. H. Jang, J. W. Yoon, K. C. Ro, N. Y. Park, and S. M. Jang, "Performance of a Brushless DC Motor due to the Axial Geometry of the Permanent Magnet," IEEE Trans. Magn., vol. 33, no. 5, pp. 4101-4103, Sep. 1997.

[9] Richard Perers, Urban Lundin, and Mats Leijon, "Saturation Effects on Unbalanced Magnetic Pull in a Hydroelectric Generator With an Eccentric Rotor," IEEE Trans. Magn., vol. 43, no. 10, pp. 3884-3890, Oct. 2007.

[10] G. H. Jang, and J. W. Yoon, "Three Dimensional Analysis of Magnetic Force and Torque in a BLDC Motor due to the Axial Variation of Yoke and Pole," IEEE Trans. Magn., vol. 35, no. 5, pp. 3694-3696, Sep. 1999.

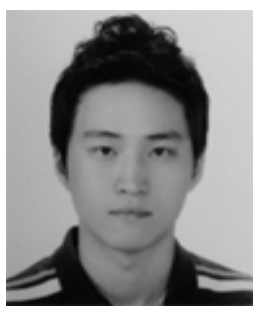

Myung-Ki Seo He was born in Suwon, Korea, in 1989. He received the B.S. degree in electronic and electrical engineering from Hongik University, Seoul, Korea, in 2014. He is currently working toward the M.S. degree in the Department of Electrical and Computer Engineering, Sungkyunkwan University, Suwon, Korea. His research interests include numerical analysis and design optimization of electric machine and power apparatus.

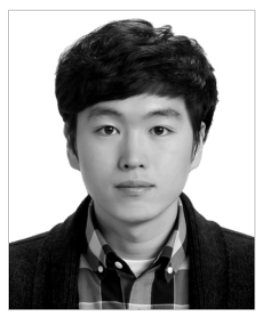

Tae-Yong LEE He was born in Suwon, Korea, in 1987. He received the B.S. degree in electrical and computer engineering from Sungkyunkwan University, Suwon, Korea, in 2013. He is currently working toward the M.S. degree in the Department of Electrical and Computer Engineering, Sungkyunkwan University, Suwon, Korea. His research interests include numerical analysis and design optimization of electric machine and power apparatus.

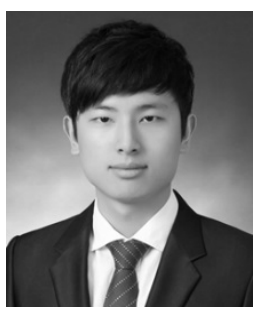

Kyungsoo Park $\mathrm{He}$ was born in Daejeon, Korea, in 1990. He received the B.S. degree in electrical and computer engineering from Sungkyunkwan University, Suwon, Korea, in 2015. He is currently working toward the M.S. degree in the Department of Electrical and Computer Engineering, Sungkyunkwan University, Suwon, Korea. His research interests include numerical analysis and design optimization of electric machine and power apparatus.

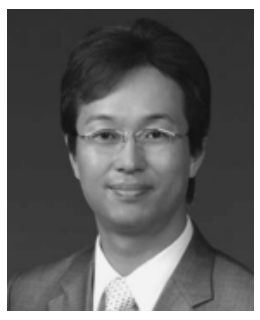

Yong-Jae Kim He was born in Gwangju, Korea, in 1973. He received the B.Eng. degree in electrical engineering from Chosun University, Gwangju, in 1996 and the M.Eng. and Dr. Eng. degrees in electrical and electronic engineering from the Musashi Institute of Technology, Tokyo, Japan, in 2003 and 2006, respectively. From 2006 to 2007, he was a Researcher of electrical and electronic engineering with the Musashi Institute of Technology. He is currently an Associate Professor with the Department of Electrical Engineering, Chosun University. His current research interests include the design and analysis of electric machines. 


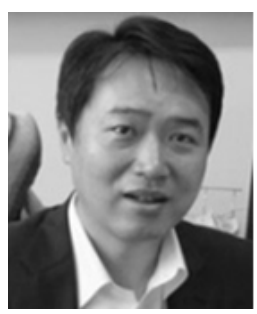

Sang-Yong Jung He received the B.S., M.S., and Ph.D. degrees in electrical engineering from Seoul National University, Seoul, Korea, in 1997, 1999, and 2003, respectively. From 2003 to 2006, he was a Senior Research Engineering with the R\&D Division, Hyundai Motor Company, Korea, and the R\&D Division, Kia Motor, Korea. From 2006 to 2011, he was an Assistant Professor with the Department of Electrical Engineering, Dong-A University, Busan, Korea. Since 2011, he has been an Associate professor with the Department of Electrical and Computer Engineering, Sungkyunkwan University, Suwon, Korea. His research interests include the numerical analysis and optimal design of electric machines and power apparatus. 